\title{
Conceptual mobility model of vertical handover decision in heterogeneous networks
}

\author{
Norakmar Arbain ${ }^{1}$, Zolidah Kasiran ${ }^{2}$ \\ ${ }^{1}$ Faculty of Electrical Engineering, Universiti Teknologi MARA (UiTM), Malaysia \\ ${ }^{2}$ Faculty of Computer Science and Mathematics, Universiti Teknologi MARA (UiTM), Malaysia
}

\begin{tabular}{l} 
Article Info \\
\hline Article history: \\
Received Oct 10, 2018 \\
Revised Dec 6, 2018 \\
Accepted Dec 20, 2018 \\
\hline
\end{tabular}

Keywords:

Heterogeneous network MADM

Mobility management

Mobility pattern

Vertical handover

\begin{abstract}
In heterogeneous network, maintaining seamless connectivity needs excessive efforts from various aspects such as network availability and mobile node reliability. Presently, a vertical handover management is a practical approach in facilitating the service continuity for mobile users. Many researches have been conducted in this area by considering performance improvement in delay, latency, and overhead. Preserving the Quality of Services (QoS) based on user mobility and pattern movement during handover decision has become an important aspect in vertical handover management. This paper presents the conceptual mobility model of vertical handover decision in heterogeneous network. Hence, several researches in vertical handover decision management has been reviewed regarding the issues on the vertical handover decision algorithms such as RSS Based Algorithm, MADM Based Algorithm and Intelligence Based Algorithm. This paper highlights the current decision algorithms that integrate the traditional methods with intelligence algorithm for better optimization. In decision parameters, the user mobility pattern can be importance in terms of direction randomness and mobility speed. Hence, a conceptual mobility-awareness model for vertical handover are been proposed in targeting some improvement of handover performance.
\end{abstract}

Copyright $@ 2019$ Institute of Advanced Engineering and Science. All rights reserved.

\section{Corresponding Author:}

Norakmar Arbain,

Faculty of Electrical Engineering, Universiti Teknologi MARA (UiTM), 40450 Shah Alam, Selangor, Malaysia.

Email: niezmar@gmail.com

\section{INTRODUCTION}

Recently, mobile devices such as smartphones, laptops and tablets have been extensively used with support of advanced network technologies. An internet access and communication activity happen at anytime and anywhere. Mobility may affect the communication connectivity when the mobile node (MN) makes changes on the preferred network during the communication process. Hence, a good approach of mobility management is essential in preserving the Quality of Service (QoS) to user. Mobility management in heterogeneous wireless network has emerged and researched over the years [1]-[3]. One of the important parts in mobility management is how to manage the handover process when the mobile node changes or move away from current service network to another service network without disrupting the communication connectivity. Handover is a process when the mobile node moves from one wireless cell to another and requires the seamless connection with the next wireless cell. A seamless connectivity [2] or "Always Best Connected" is about maintaining the network connectivity of all running applications on the mobile device. This is still a challenging task to facilitate the best approach that can support the seamless connectivity while maintaining the QoS at user levels [3]. 
Handover management in wireless network can be carried out either horizontally or vertically. Horizontal handover happened in homogeneous environment where the network changing process occurred within the same domain of network, such as the mobile node changed the preferred network from WLAN1 to WLAN2 within the same domain. Meanwhile, in heterogeneous network, vertical handover is more challenging as the mobile node needs to change the preferred network from different network domain and technologies, such as WiMax, WiFi, UMTS/LTE and others. Moreover, the handover management procedure can be classified into three types which are hard, soft and softer handover. Hard handover is referred to breaking the connected network before make the next network connection, as the mobile node only connected to one point of attachment (POA) at a time. Meanwhile, soft handover allowed mobile node to connect to two POA for a while until the best connection obtained. However, softer handover [4] is the best approach in handover management where this fast and smooth handover can minimise the latency and packet loss during handover execution. In general, Handover Management Architecture consists of three parts which are Handover Initiation, Handover Decision and Handover Execution.

\subsection{Handover Initiation}

During system discovery, all information from mobile node and networks are collected such as battery power, network bandwidth and signal strength. QoS requirements are also taken into consideration as an input for the next handover phase.

\subsection{Handover Decision}

At this phase, a mobile node needs to make a new network selection because of some factors such as rapid mobile node movement and stumpy network coverage. Mainly, handover decision selects new preferred network based on the received signal strength and highest-ranking indicator collected from system discovery process. Some decision algorithms are manipulated using mathematical or computational formula for managing the handover performance like overhead and delay.

\subsection{Handover Execution}

When the selected network satisfies the QoS requirements of mobile node, the handover executed based on the certain handover controller. The handover management controller can be either at mobile node or network side. Hence, four types of handover process control are Network Controlled HandOver (NCHO), Mobile Controlled HandOver (MCHO), Mobile-Assisted HandOver (MAHO) and Network-Assisted HandOver (NAHO) [2].

This paper presents the conceptual mobility model of vertical handover decision in heterogeneous network and organized as follows. Section 2 discussed the current vertical handover management including the vertical handover decision algorithms analysis. Section 3 presents the proposed mobility-awareness model during network selection. Section 4 encompasses the conclusion and future recommendations on this research.

\section{RESEARCH METHOD}

\subsection{Vertical Handover Decision (VHD) Algorithm}

Several literatures have presented an overview of vertical handover decision strategies in different categories. In research [5], the authors make the comparison between vertical handover decision strategies in five categories such as Decision Function (DF), User Centric (UC), Multiple Attribute Decision (MAD), Fuzzy Logic/Neural Network (FL/NN) and Context-Aware (CA). They proposed a new handover decision scheme contains two components which are Fuzzy Logic System (FLS) and Network Selection (using AHP method). Authors in [6], propose a vertical mobility management architecture named as Context-Aware Mobility Management System (CAMMS) which support the cross-layer, context-aware and seamless handover for user and services. They design four main components of functional entities that responsible for context gathering, intelligent handover decision-making, accurate handover triggering and post-handoff management.

J. Márquez-Barja et al. [7], review on algorithms, protocols and tools in vertical handover management. The paper focus on vehicular network, where vehicle as a node that have several constrained such as topology restrictions, mobility patterns, power consumption, scalability, reliability and speed. Research in [8] categorized the VHO Algorithm into four types which are Location Based Handover, Mobility Based Handover, Policy Based Handover and Learning Based Handover. Meanwhile, authors in [9] conducted a survey of handover decision algorithms for LTE-A femtocells. They classified the HO Algorithm into five aspect which are RSS, Speed, Interference-aware, Cost-function and Energy-efficient. They also highlighted on the need of future research on multiple-macrocell multiple-femtocell scenario where RSS and 
RSQ as main parameters. Yan et al. in [10], summarize the vertical handover decision algorithm into four categories which are RSS Based VHD Algorithms, Bandwidth Based VHD Algorithms, Cost Function Based VHD Algorithms and Combination Based VHD Algorithms. Twelve VHD algorithms have been analyzed and they presented the advantages and disadvantages of each algorithm. The current vertical handover decision can be categories into RSS based Algorithm, MADM based Algorithm and Intelligence based Algorithm as shown in Figure 1.

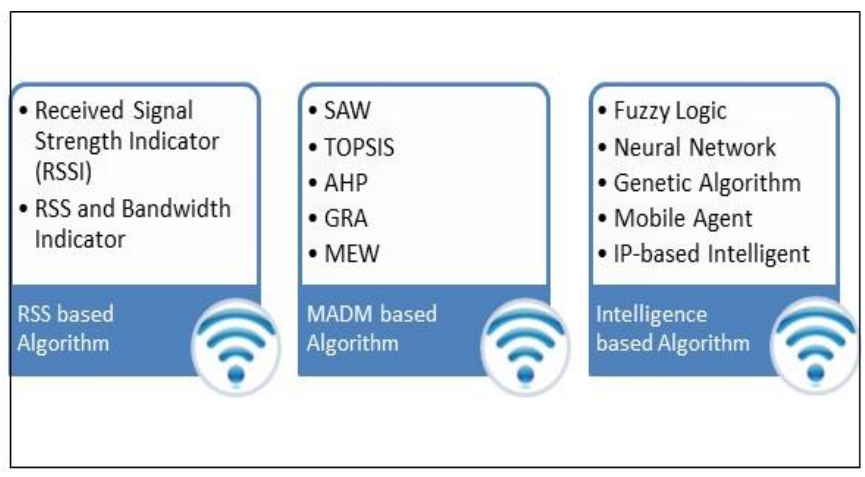

Figure 1. Vertical handover decision schemes

\subsubsection{RSS based Algorithm}

Received Signal Strength (RSS) is a common handover decision. In [11], a mathematical model based on VHO prediction approach has been review by considering some parameters such as RSS, UE velocity, load and cost per user bandwidth. The proposed algorithm has been simulated in Matlab and follows the Jake's model. Evaluation of the network performance is based on user velocity and handover numbers. Handover process evaluated in WiFi and WiMax access networks. Authors in [12]-[13], evaluate the handover performance based on Received Signal Strength Indicator (RSSI) algorithm for WLAN/Cellular network for Mobile Voice users. Ning et al. in [12], proposed handover algorithm based on RSS and Markov mobility model for minimizing the number of handovers.

\subsubsection{MADM based Algorithm}

Multiple Attribute Decision Making (MADM) based algorithm is a popular decision method. MADM methods can facilitate the need of multi-criteria solution for avoiding inappropriate handover decision as highlights in [5]. In research [13], Maaloul et al. proposed an efficient handover decision algorithm based on MADM method. The paper highlights some MADM methods such as Simple Additive Weighting (SAW), Weight Product Method (WPM), Technique for Order Preference by Similarity to Ideal Solution (TOPSIS), Grey Relational Analysis (GRA), Distance to Ideal Alternative (DIA), VIKOR and ELECTRE. They proposed a Ranking Scheme based on provided QoS algorithm where considering contextawareness in the evaluation parameters like coverage area, RSSI, available bandwidth, delay, response time, jitter, security, user preference and cost. This research compared the proposed algorithm with some MADM methods mention earlier. Result shows some improvement in handover performance; however, the algorithm complexity may increase the handover delay and overhead.

In [14], a network selection based on context awareness services has been proposed by the authors. This context-awareness algorithm combines the fuzzy mathematics evaluation and TOPSIS-MADM approach. From their simulation results, fuzzy mathematics evaluation has performed better regards to network selection speed. The TOPSIS-MADM approach supports well in utility performance measurement. Authors in [15] compared handover performance for four MADM based vertical handover algorithm namely MEW (Multiplicative Exponent Weighting), SAW, TOPSIS and GRA. Based on their simulation result, GRA has slightly higher bandwidth and lower delay value when concerning the weight of attributes such as bandwidth, delay, jitter and bit error rate (BER). In [16], the research presented the handover performance evaluation on seven MADM methods which are SAW, MEW, TOPSIS, GRA, ELECTRE, VIKOR and WMC. The numerical simulation is designed in MATLAB software under means of different application scenarios such as voice, data and cost-constrained connection. The outcome shows that SAW and GRA has low computational complexity and a possible solution for seamless vertical handover. Meanwhile, when considering the types of applications like voice and data, VIKOR and MEW are the best MADM decision methods.

Conceptual mobility model of vertical handover decision in heterogeneous networks (Norakmar Arbain) 


\subsubsection{Intelligence based Algorithm}

Traditional RSS and MADM based algorithm still have some limitations when applied in heterogeneous networks. These methods create only small difference values in ranking accuracy [17] and fixed network discovery method [18]. Hence, combining the traditional methods with intelligence-based algorithm may leads to better handover performance. Authors in [18] works on an type-2 fuzzy logic algorithm that specifically focused on vehicular heterogeneous networks. Pink et al. in [19] presented a fuzzybased vertical handover decision algorithm which can adapts with device and network capabilities. The research has been experimented in real environment with controlled device which considering the UMTS/WLAN networks. The evaluation focused on QoS and resource consumption. Result show that mobility affects the QoS as the mobile node changes the path, the signal strength may changes accordingly. In minimising the resource consumption, the algorithm slightly decrease the maximum device runtime up.

Research in [20] proposed a new hybrid algorithm technique using combination of ABC (Ant Bee Colony) and PSO (Particle Swarm Optimization in the process of selecting the best wireless network. Meanwhile, authors in [21] develops a model for handover decision algorithm by applying hybrid Artificial Neural Network (ANN). Both approaches able to reduce the cost and ping-pong effects in handover. Luo et al. [22], proposed a new handover prediction algorithm based on Hidden Markov Model. The experiment conducted for wireless network in office environment by considering the controlled situation. Hence, the user's mobility in predictable and RSS value is accurately measured. Still, the research has limitation on the mobility model and emission probability need to know and learns on each other better. Table 1 show the summary of current vertical handovers decision algorithms.

Table 1. Summary of Vertical Handovers Decision Algorithms

\begin{tabular}{|c|c|c|c|}
\hline & RSS & MADM & Intelligence Algorithms \\
\hline Review papers & \multicolumn{3}{|c|}{$\begin{array}{l}\text { Survey and review papers on mobility management } \\
{[5]-[10],[23]}\end{array}$} \\
\hline Basic - RSS & $\begin{array}{l}\text { Received Signal Strength } \\
{[11]-[12],[24]-[25]}\end{array}$ & $\begin{array}{l}\text { Ranking Scheme [13] } \\
\text { MEW, SAW, TOPSIS, GRA [15] } \\
\text { SAW, MEW, TOPSIS, GRA, } \\
\text { ELECTRE, VIKOR, WMC [16] }\end{array}$ & NA \\
\hline Fuzzy Logic (FL) & $\begin{array}{l}\text { RSS, Fuzzy [26] } \\
\text { Fuzzy[27] }\end{array}$ & $\begin{array}{l}\text { MCDM Fuzzy-AHP [3] } \\
\text { Fuzzy-TOPSIS [14] } \\
\text { Fuzzy-AHP [28] } \\
\text { MCDMFuzzy-TOPSIS [29] } \\
\text { Fuzzy MADM [30] } \\
\text { MADM - GRA [31] }\end{array}$ & $\begin{array}{c}\text { Fuzzy-MADM [17] } \\
\text { Type-2 Fuzzy [18] } \\
\text { Fuzzy [19] }\end{array}$ \\
\hline $\begin{array}{c}\text { Artificial Neural Network } \\
\text { (ANN) }\end{array}$ & NA & NA & ANN [21] \\
\hline Ant Bee Colony (ABC) & NA & NA & $\begin{array}{l}\text { Cloud Assisted Handover } \\
\text { (IoT) - ABC [32] }\end{array}$ \\
\hline $\begin{array}{l}\text { Particle Swam Optimization } \\
\text { (PSO) }\end{array}$ & NA & NA & $\begin{array}{l}\text { PSO-ABC [20] } \\
\text { PSO [33] }\end{array}$ \\
\hline Markov Chain & MDP [34] & NA & Hidden Markov [22] \\
\hline MIH \& SDN & MIH [35] & VIKOR [36] & NA \\
\hline
\end{tabular}

Note: NA - Not Applicable

Research in [37] focus on distributed handover and mobility pattern prediction by using Markov theory and statistical theory. They proposed an algorithm named Pattern Prediction and Passive Bandwidth Management Algorithm (3P-BMA) which apply Markovian Prediction scheme. Yet, this research only focused on guarantees the service continuity in wireless cellular networks. Authors in [38] also focused on mobility prediction for wireless cellular network, in optimizing the Call Admission Control (CAC) by proposed the In-advance Multiplexing Call Admission Control (IAM-CAC) scheme. This new CAC scheme based on threshold approach has been perform wells in terms of Call Blocking Probabilities/Call Dropping Probabilities.

In addition, authors in [39], focused on link-layer inter-technology handovers, in supporting the limitations of user mobility randomness, high handover overhead, optimality requirement. The paper proposed a new handover decision making that exploits the data traces of user mobility in real mobile environments. They are mining the data from Dartmouth College, a campus environment for assuming the strong regularity with user mobility. The decision process was formulates using Markov decision process (MDP) and employs using MADM-AHP approach. The algorithm has been compared with random and greedy algorithm, with the results that shown the new algorithm performs better. However, this method requires extensive data traces and simulation scenarios. 


\subsection{Proposed Methods}

The proposed scenarios for vertical handover are shown in Figure 2. A few scenarios will be setup for collecting the human mobility pattern. The sample data will be collected from several mobile nodes in campus network environment. The data will be identified using Livelab network measuring source code. The chosen access technologies will be a WLAN (WiFi:IEEE 802.11) and a LTE network. The designed mobility pattern model will be included in vertical handover framework.

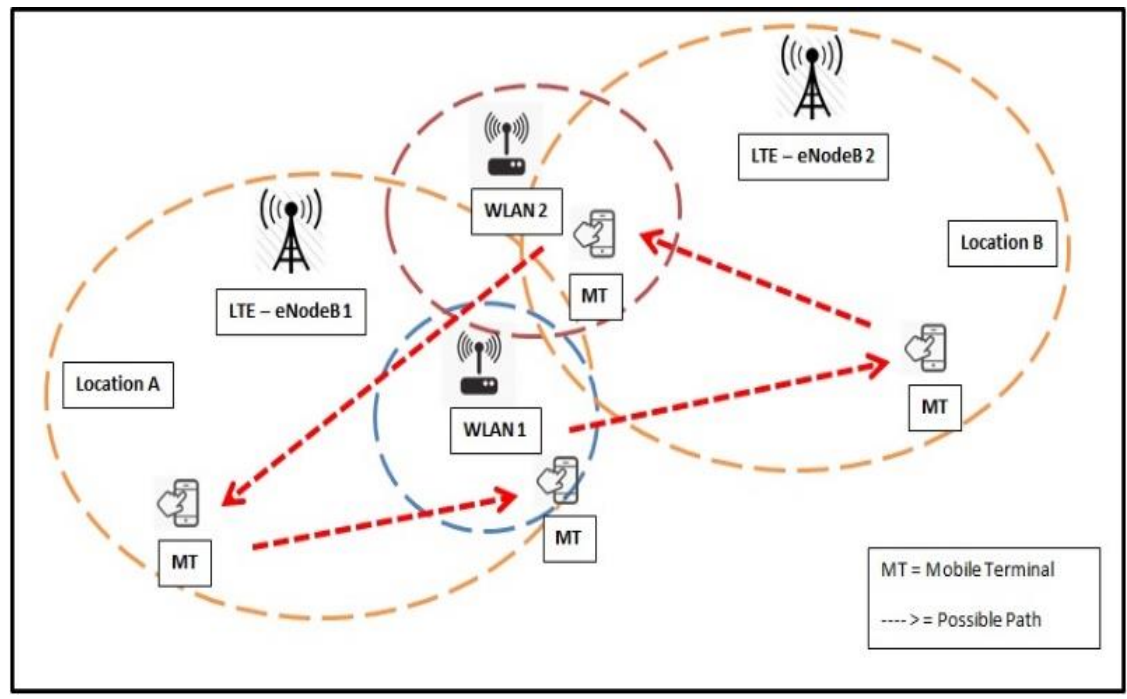

Figure 2. Proposed scenarios for vertical handover

The proposed scenarios will refer to research in [40] as shown in Table 2. The collected data will be measured and analyzed for respected parameters such as user's movement pattern, speed and location. From this real data traces, a pattern model will be construct and used in latter proposed algorithm. A sample data from online database named CRAWDAD also will be used as benchmark value in comparison with the real data traces collected in Livelab Networks. In addition, a crowdsourcing application named as OpenSignal will be used for collecting and retrieving network performance statistics, at target location respectively. This application can records the performance statistics of connections between mobile nodes with the eNodeB (LTE) and WLAN (WiFi). It also provides an aggregated statistics of network coverage that presented in the form of coverage maps.

Table 2. Scenarios for Collecting Online Mobility Pattern

\begin{tabular}{cccc}
\hline Scenarios & Network Selection & Data Technologies & Applications \\
\hline Case 1 & LTE to WiFi & General/Conventional & Web Browser, Voice, SNS \\
Case 2 & WiFi to LTE & General/Conventional & \\
Case 3 & LTE to WiFi & Streaming & Video, Live TV \\
Case 4 & WiFi to LTE & Streaming & \\
\hline
\end{tabular}

\section{RESULTS AND ANALYSIS}

\subsection{Random Mobility Model and Algorithm}

In recent years, several researches began focusing on the mobility issue as mobile node may have different mobility speed and movement direction. Hence, considering the randomness of mobile node's movements and variety of mobility speed is still a challenging task in vertical handover management. Commonly, main parameters in vertical handover decision are based on network conditions such as received signal strength and available bandwidth. Besides, at the user side (mobile node), several conditions also being considered like mobile energy, connection cost and user preferences. Table 3 show the parameter metrics for Random Mobility Parameters in Mobility-awareness of Vertical Handover Management for LTE-WLAN networks. Meanwhile, a block diagram in Figure 3 show the Fuzzy-Topsis with Mobility-awareness model. 
Table 3. Random Mobility Parameters in Mobility-awareness of Vertical Handover Management

\begin{tabular}{cccc}
\hline Parameter & Indication Value & Parameter & Indication Value \\
\hline Received Signal Strength & Rss & MT Move Out & MTmo \\
Available Bandwidth & bd & Speed & s(n) \\
MT Move In & MTmi & Directions & d(n)
\end{tabular}

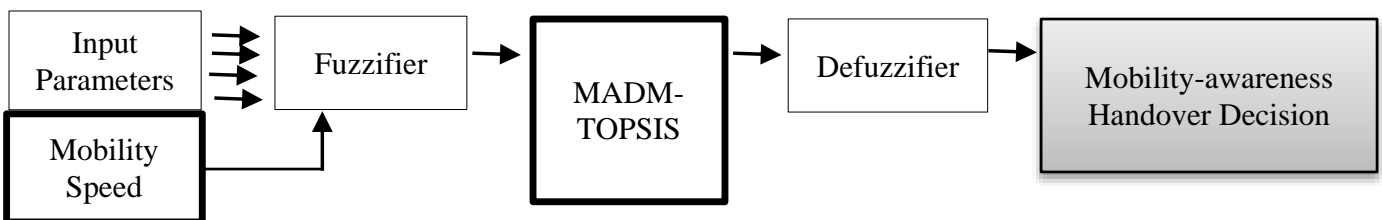

Figure 3. Fuzzy-TOPSIS block diagram with random mobility-awareness

\subsection{Network Performance and Availability}

Figure 4 show the network performance statistics, at targeted location respectively based on a crowdsourcing application named as OpenSignal. This application can record the performance statistics of connections between mobile nodes with the eNodeB (LTE) and WLAN (WiFi). It also provides an aggregated statistics of network coverage that presented in the form of coverage maps.

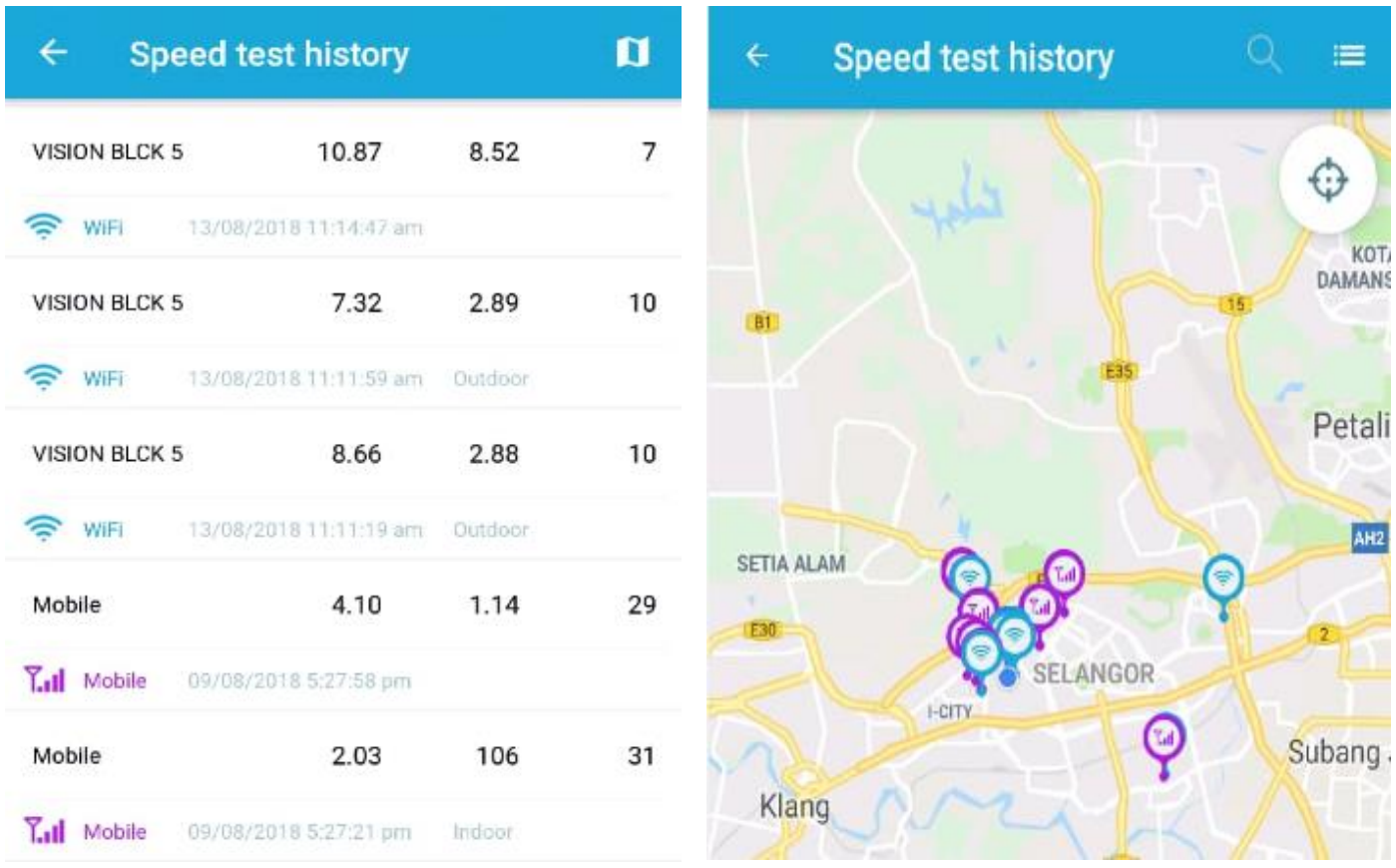

Figure 4. Network performance speed test history and coverage maps from OpenSignal Apps

\subsection{Conceptual Model of Mobility-awareness Vertical Handover Management}

Relatively, a proposed new vertical handover decision that exploits mobility-awareness model should consider the mobile node's speed and pattern randomness as shown in Figure 5. 


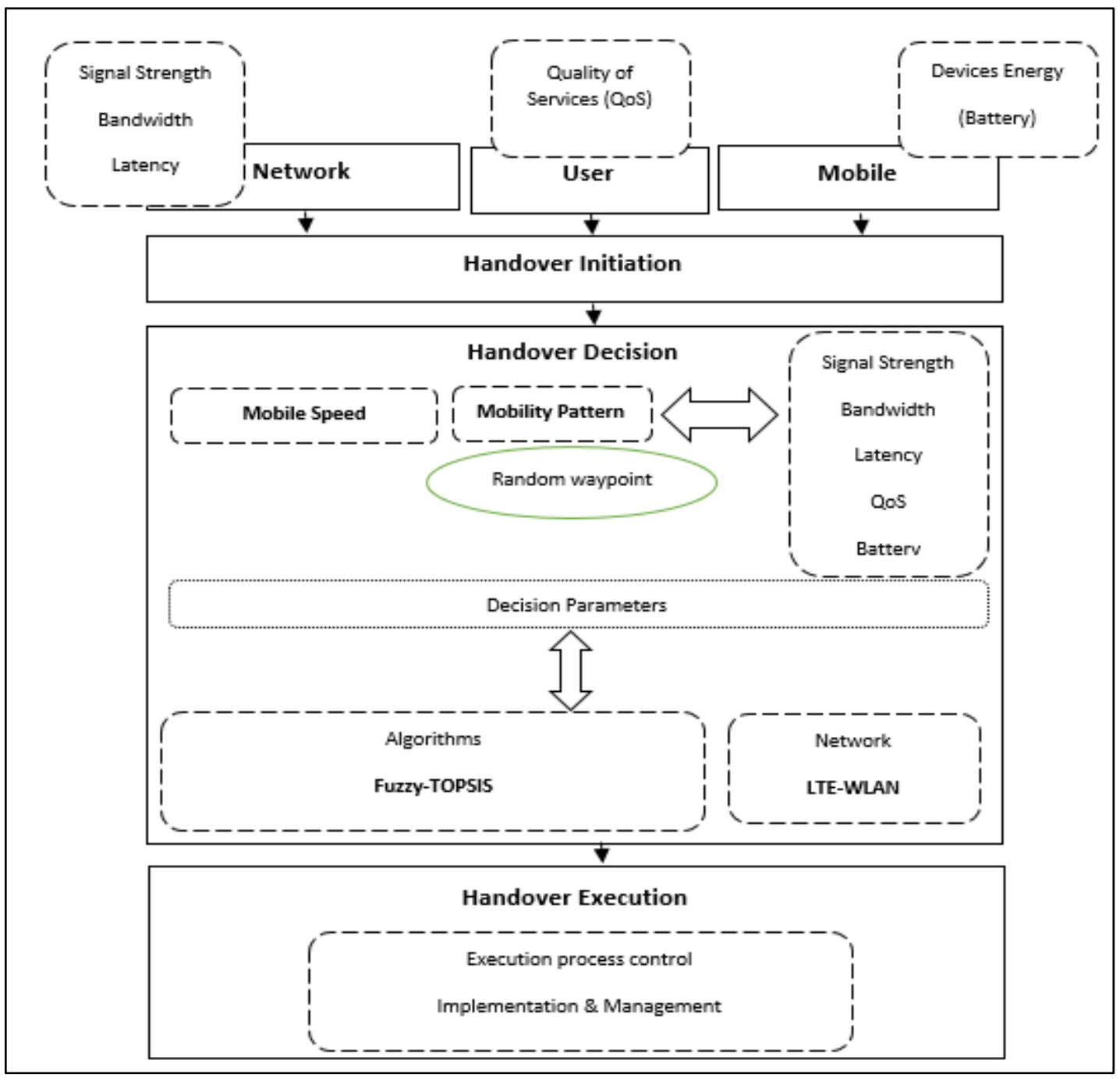

Figure 5. Mobility-awareness vertical handover management

\section{CONCLUSION}

This paper presents the conceptual mobility model of vertical handover decision in heterogeneous network. Hence, maintaining network connectivity in heterogeneous network requires astounding effort in academia and industries. Rapid mobility has become a bigger challenge in facilitating the service continuity to mobile users. Furthermore, variety of network access such as WiFi, WiMAX, 3GPP and LTE also creates chaotic mobile environments when the handover process not being conducted precisely. Hence, this paper reviews several current researches on vertical handover management architecture. In addition, the literatures on vertical handover decision algorithm also being analyse and categorised into three sections which are RSS Based Algorithm, MADM Based Algorithm and Intelligence Based Algorithm. Most current researches focused on combining the RSS and MADM approaches with intelligence algorithm like Fuzzy logic, to enhance the handover performance such as overhead and delay. From the related reviews, this paper proposed a mobility-awareness vertical handover management that encompasses mobility speed and pattern as an important attribute during the handover decision process. Hence, we hope the future vertical handover management can provide more seamless network connectivity.

\section{ACKNOWLEDGEMENTS}

This research was financially supported by the Ministry of Higher Education (MOHE) through Fundamental Research Grant Scheme (FRGS), 600-RMI/FRGS 5/3(0006/2016). 


\section{REFERENCES}

[1] N. Omheni, et al., "Smart mobility management in 5G heterogeneous networks," IET Networks, vol/issue: 7(3), pp. 119-128, 2018.

[2] A. Gani, et al., "A review on interworking and mobility techniques for seamless connectivity in mobile cloud computing," J. Netw. Comput. Appl., vol. 43, pp. 84-102, 2014.

[3] M. Drissi, et al., "A Multi-Criteria Decision Framework for network selection over LTE and WLAN," Eng. Appl. Artif. Intell., vol. 66, pp. 113-127, 2017.

[4] N. K. Panigrahy and S. C. Ghosh, "Analyzing the Effect of Soft Handover on Handover Load Condition," IEEE Trans. Veh. Technol., vol/issue: 67(4), pp. 3612-3624, 2018.

[5] M. Kassar, et al., "An overview of vertical handover decision strategies in heterogeneous wireless networks," Comput. Commun., vol/issue: 31(10), pp. 2607-2620, 2008.

[6] S. Fernandes and A. Karmouch, "Vertical Mobility Management Architectures in Wireless Networks: A Comprehensive Survey and Future Directions," IEEE Commun. Surv. Tutorials, vol/issue: 14(1), pp. 45-63, 2012.

[7] J. M. Barja, et al, "An overview of vertical handover techniques: Algorithms, protocols and tools," Comput. Commun., vol/issue: 34(8), pp. 985-997, 2011.

[8] G. Gódor, et al., "A survey of handover management in LTE-based multi-tier femtocell networks: Requirements, challenges and solutions," Comput. Networks, vol. 76, pp. 17-41, 2015.

[9] D. Xenakis, et al., "Mobility management for femtocells in LTE-advanced: Key aspects and survey of handover decision algorithms," IEEE Commun. Surv. Tutorials, vol/issue: 16(1), pp. 64-91, 2014.

[10] X. Yan, et al., "A survey of vertical handover decision algorithms in Fourth Generation heterogeneous wireless networks," Comput. Networks, vol/issue: 54(11), pp. 1848-1863, 2010.

[11] S. B. Johnson, et al., "An Optimized Algorithm for Vertical Handoff in Heterogeneous Wireless Networks," IEEE Conf. Inf. Commun. Technol., pp. 1206-1210, 2013.

[12] Z. Ning, et al., "Markov-based vertical handoff decision algorithms in heterogeneous wireless networks," Comput. Electr. Eng., vol/issue: 40(2), pp. 456-472, 2014.

[13] S. Maaloul, et al., "An efficient handover decision making for heterogeneous wireless connectivity management," 201321 st Int. Conf. Software, Telecommun. Comput. Networks - (SoftCOM 2013), pp. 1-8, 2013.

[14] L. U. O. Lijuan, et al., "Network Selection Based on Context-Awareness Services," China Commun., pp. 50-56, 2013.

[15] E. S. Navarro and V. W. Wong, "Comparison between Vertical Handoff Decision Algorithms for Heterogeneous Wireless Network," Veh. Technol. Conf., vol. 2, pp. 947-951, 2006.

[16] E. S. Navarro, et al., "Evaluation of Vertical Handoff Decision Algorightms Based on MADM Methods for Heterogeneous Wireless Networks," J. Appl. Res. Technol., vol. 10, pp. 534-548, 2012.

[17] H. Jin, et al., "IFMADM: An efficient network selection algorithm in integrated heterogeneous wireless network," 2011 4th IEEE Int. Conf. Broadband Netw. Multimed. Technol., pp. 511-516, 2011.

[18] B. Ma and X. Liao, "Speed-adaptive vertical handoff algorithm based on fuzzy logic in vehicular heterogeneous networks," 2012 9th Int. Conf. Fuzzy Syst. Knowl. Discov., pp. 371-375, 2012.

[19] M. Pink, et al., "Towards a seamless mobility solution for the real world: Handover decision," 2012 Int. Symp. Wirel. Commun. Syst., pp. 651-655, 2012.

[20] S. Goudarzi, et al., "ABC-PSO for vertical handover in heterogeneous wireless networks," Neurocomputing, vol. 256, pp. 63-81, 2017.

[21] A. M. Aibinu, et al., "Development of hybrid artificial intelligent based handover decision algorithm," Eng. Sci. Technol. an Int. J., vol/issue: 20(2), pp. 381-390, 2017.

[22] Y. Luo, et al., "Handover prediction for wireless networks in office environments using Hidden Markov Model," 2013 IFIP Wirel. Days, pp. 1-7, 2013.

[23] J. Wu and P. Fan, "A Survey on High Mobility Wireless Communications: Challenges, Opportunities and Solutions," IEEE Access, vol. 4, pp. 450-476, 2016.

[24] T. Ali and M. Saquib, "Performance Evaluation of WLAN/Cellular Media Access for Mobile Voice Users under Random Mobility Models,” IEEE Trans. Wirel. Commun., vol/issue: 10(10), pp. 3241-3255, 2011.

[25] T. Ali and M. Saquib, "Analytical framework for WLAN-cellular voice handover evaluation," IEEE Trans. Mob. Comput., vol/issue: 12(3), pp. 447-460, 2013.

[26] X. S. and J. Z. L. Zhang, L. Ge, "Fuzzy Logic based Vertical Handover Algorithm," 017 26th Wireless and Optical Communication Conference (WOCC), 2017.

[27] J. M. Kang, et al., "Autonomic personalized handover decisions for mobile services in heterogeneous wireless networks," Comput. Networks, vol/issue: 55(7), pp. 1520-1532, 2011.

[28] R. K. Goyal, et al., "The utility based non-linear fuzzy AHP optimization model for network selection in heterogeneous wireless networks," Appl. Soft Comput. J., 2016.

[29] Y. S. Hussein, et al., "A novel cell-selection optimization handover for long-term evolution (LTE) macrocellusing fuzzy TOPSIS,” Comput. Commun., vol. 73, pp. 22-33, 2016.

[30] A. B. Zineb, et al., "An Enhanced Vertical Handover Based on Fuzzy Inference MADM Approach for Heterogeneous Networks,” Arab. J. Sci. Eng., vol/issue: 42(8), pp. 3263-3274, 2017.

[31] M. Mansouri and C. Leghris, "A battery level aware MADM combination for the vertical handover decision making," 2017 13th Int. Wirel. Commun. Mob. Comput. Conf. IWCMC 2017, pp. 1448-1452, 2017.

[32] D. Li, et al., "A cloud-assisted handover optimization strategy for mobile nodes in industrial wireless networks," Comput. Networks, vol. 128, 2017. 
[33] K. Ahuja, et al., "Particle swarm optimization based network selection in heterogeneous wireless environment," Opt. - Int. J. Light Electron Opt., vol/issue: 125(1), pp. 214-219, 2014.

[34] S. Zang, et al., "Mobility handover optimization in millimeter wave heterogeneous networks," in 17th International Symposium on Communications and Information Technologies, ISCIT 2017, pp. 1-6, 2017.

[35] W. Mansouri, et al., "Cross layer architecture with integrated MIH in heterogeneous wireless networks," Comput. Networks, vol. 127, pp. 126-137, 2017.

[36] X. Li, et al., "A novel optimized vertical handover framework for seamless networking integration in cyber-enabled systems," Futur. Gener. Comput. Syst., vol. 79, pp. 417-430, 2016.

[37] P. Fazio, et al., "A distributed hand-over management and pattern prediction algorithm for wireless networks With mobile hosts," 9th International Wireless Communications and Mobile Computing Conference, IWCMC 2013, pp. 294-298, 2013.

[38] P. Fazio, et al., "Mobility prediction in wireless cellular networks for the optimization of Call Admission Control schemes," 2014 IEEE 27th Can. Conf. Electr. Comput. Eng., pp. 1-5, 2014.

[39] Y. Zhu, et al., "Exploiting mobility patterns for inter-technology handover in mobile environments," Comput. Commun., vol/issue: 36(2), pp. 203-210, 2013.

[40] M. Lahby, et al., "An enhanced-TOPSIS based network selection technique for next generation wireless networks," Int. Conf. Telecommun., pp. 1-5, 2013.

\section{BIOGRAPHIES OF AUTHORS}

\begin{tabular}{|l|l|} 
Norakmar Arbain @ Sulaiman received her B.Sc. degree in Information System Engineering \\
(Computer Science) in 2005, her M.Sc. in Real Time Software Engineering in 2009, while she is \\
currently pursued her Ph.D. at the Faculty of Computer Science and Mathematics, at Universiti \\
Teknologi MARA, Malaysia. Norakmar is also a lecturer at Faculty of Electrical Engineering of \\
Universiti Teknologi MARA. Her current research interests are in the areas of mobile \\
networking, cloud computing and software engineering.
\end{tabular}

